\title{
Research of Workflow nets Application Based on the Petri Nets
}

\author{
Qi Dabin \\ University of Science and Technology \\ Liaoning, \\ Anshan, China, 13610981503 \\ qdbinner@163.com
}

\author{
Yao Jun \\ Chemical plant of Ansteel stock \\ company, \\ Anshan China
}

\author{
Li Qiuju \\ Anshan Normal University Liaoning \\ China \\ Anshan , China
}

\begin{abstract}
Abstrac-This paper introduced basic knowledge of Petri nets and workflow, and invariant analysis technique of Petri nets models structure, and integrate the real application circumstances of the workflow of receive document in document management, constructs an integrated workflow model based on Petri net. Further more, the author utilize invariant analysis technique to provide the verification of the model.
\end{abstract}

Key words-Petri Nets; Workflow nets; Performance analysis; Receive document

In recent years, Petri-net theories developed concurreny、 synchrony, enlogy and net topology as primary content . Petrinet application has refer to every field of computer science , especially, workflow application field . Petri- net refered to the better maths base and visual modeling . and it has become implement of workflow study on analyzing and modeling.

\section{I .PETRI-NET DEFINITION AND FUNCTION RULE.}

Petri-net definition: Petri-net is defined as four-dimension groups $\mathrm{PN}(\mathrm{P}, \mathrm{T}, \mathrm{F}, \mathrm{M} 0), \mathrm{P}$ is named place sets, $\mathrm{P}=(\mathrm{P} 1, \mathrm{P} 2, \cdots)$ is not full sets. $\mathrm{T}$ is named transition sets, $\mathrm{T}=(\mathrm{T} 1, \mathrm{~T} 2, \ldots)$ is not full sets.F is flow relation, M0 is initial marking. In Petri-net, "O" denotes a place (middle state); rectangle denotes a transition ; the arrowhead from $\mathrm{P}$ to $\mathrm{T}$ ( or from $\mathrm{T}$ to $\mathrm{P}$ ) denotes a arc in flow relation.

Dynamic action is denoted by token, namely symbol " •" in Petri-net. $\mathrm{T}$ is activated by condition which token amounts from $\mathrm{T}$ to $\mathrm{P}$ are greater than arc amounts from $\mathrm{P}$ to $\mathrm{T}$, namely " $t$ " has occurring authority in " $M$ " : for all " $p \in P, p$ $\in *_{t}$ ” , $M(p) \geqslant W(p, t), \quad$ “ $M$ ” enables “t” to occur, "*t" is " $\mathrm{t}$ " input sets. $\mathrm{W}(\mathrm{p}, \mathrm{t})$ is arc amounts from $\mathrm{P}$ to $\mathrm{T}$. If $\mathrm{T}$ is activated, token distribution will occur to alter in Petri-net, token amounts with transition will reduce in input place, but token amounts will increase in its output place ( " $t$ *"). " $M$ " makes " $t$ " become new marking $M^{\prime}$ ( successor marking): for all " $\mathrm{p} \in \mathrm{P}$,

$$
M^{\prime}(p)=\left\{\begin{array}{cc}
M(p)-W(p, t) & p \in{ }^{*} t \\
M(p)+W(t, p) & p \in t^{*} \\
M(p)-W(p, t)+W(t, p) & p \in^{*} t \cap t^{*} \\
M(P) & p \notin^{*} t \cap t^{*}
\end{array}\right.
$$

\section{II . WORKFLOW-NET BASED ON PETRI-NET}

Workflow model transforms Petri-net, constructing Workflow-net relying on Petri-net instrument, accomplishing Workflow-net model to evaluate.

\section{A..1 Workflow net definition}

Definition 1:

One Petri-net is Workflow-net, $\mathrm{W}=(\mathrm{P}, \mathrm{T}, \mathrm{F})$, iff:

(1) existing two places in W: place $i$ is input place, $i=\phi$; place $o$ is output place, $o^{\prime}=\phi$.

(2) Increasing a transition $t^{*}$ between Place $o$ and $i$, (namely $t^{*}=\{0\}$ and $t^{*}=\{i\}$ ), then $w^{\prime}$ is a strong connectivity Petri-net.

Definition 2:

One Petri-net is reliable, iff:

(1) for any a reachable state from $i$ to $M$, existing a executing sequence $M$ which reaching state $o, i$ is initial state and $o$ is terminative state. Namely : $\forall M(i \stackrel{*}{\longrightarrow} M) \Rightarrow M \stackrel{*}{\longrightarrow} O$

(2) for any a reachable state from $i$ to $M$, if existing a token in $o$ at least, the state is terminative state, now , the Workflow-net only has a token in $o$, other places $\begin{array}{lll}\text { should } \quad \text { be } \quad \text { empty. } & \text { Namely : } \\ \forall M(i \stackrel{*}{\longrightarrow} M \wedge M \geq 0) \Rightarrow(M=o) & \end{array}$

(3) no existing deadlock in $(w, i)$, then Petri-net is flexible. Namely: $\quad \forall t \in T, \exists M, M^{\prime}$, then $\left(i \stackrel{*}{\longrightarrow} M \stackrel{*}{\longrightarrow} M^{\prime}\right)$

B. Basic structure on Workflow net

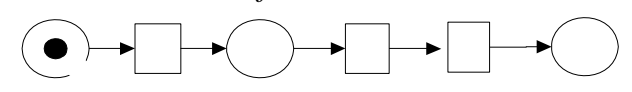

Figure 1 Serial structure

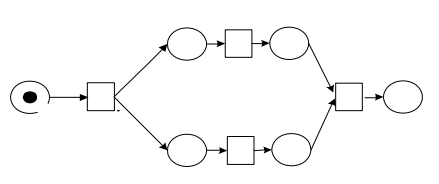

Figure 2 Parallel structure 


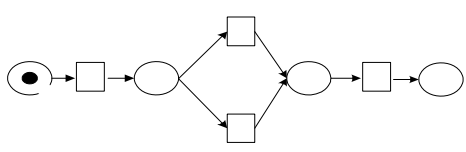

Figure 3 Choosing structure

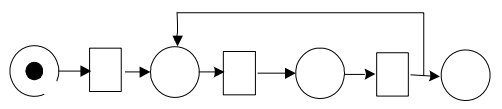

Figure 4 Circulating structure

\section{III.ANALYSIS METHOD THEORY BASED ON INVARIANT}

The method uses algebra calculation to transact system, and supposes basic net of Petri-net is simplex, namely $\forall p \in P,{ }^{*} p \cap p^{*}=\phi$.T invariant explains that marking will resume initial state afte transition sets occur, $\mathrm{T}$ invariant reflects transition sets making state return, utilizing it to study periodicity performance, etc; $\mathrm{P}_{-}$invariant reflects one weighted conservation on all token amounts in part places, utilizing it to study mutex 、 dead lock and error detection, etc.

In Petri-net analysis technology, it has great merit for Petri-net to apply invariant analysis method to confirm Petri-net performance based on simple linearity algebra equation.

\section{A..Petri-net state equation.}

In Petri-net, mk denotes Petri-net marking after running $\mathrm{k}(\mathrm{k} \geqslant 0)$ time, then the marking after running “ $\mathrm{k}+1$ ” times is:

$$
m_{k+1}=m_{k}+C u_{k}, k \geq 0
$$

$\mathrm{C}$ is incidence matrix, $\mathrm{uk}$ is activated count vector, it is one " $\mathrm{m} \times 1$ " vector, the $\mathrm{i}$ element denotes activated times by transition ti in running " $k+1$ " , formula (1) is named Petri-net state equation. All tokens in place is not negative, so running legally will ensure : $m_{k}+C u_{k} \geq 0$, for all $k \geq 0$

\section{B. $P_{-}$invariant and $T_{-}$invariant}

\section{I ) $P_{-}$invariant}

One $\mathrm{P}_{-}$invariant is one " $\mathrm{n} \times 1$ " vector $\mathrm{x}(\mathrm{x} \geqslant 0)$, and meets condition:

$$
x^{T} C=0
$$

left multiply " $\mathrm{x}^{\mathrm{T}}$ " both sides in formula (1) : $x^{T} m_{k+1}=x^{T} m_{k}+x^{T} C u_{k}$

$$
x^{T} m_{k+1}=x^{T} m_{k}, k \geq 0
$$

Beginning on deducing 'gradually from “ $\mathrm{k}=0$ " : $x^{T} m_{0}=x^{T} m_{1}=x^{T} m_{2}=x^{T} m=$ constant

$$
x^{T} m=x^{T} m_{0}=\text { constant }
$$

Formula (4) denotes the sum of initial tokens which all weighted places by $\mathrm{p}_{\text {_ invariant }}$ are constant, namely the sum of weighted tokens in all places from “ $\mathrm{m} 0$ " to reachable
" $\mathrm{m}$ " are constant, the process is called that the places are covered with $\mathrm{p} \_$invariant.

Based on incidence matrix, utilizing $p$ invariant to analyze limit and conservation performance in Petri-net structure:

(1) supposes it has only one " $\mathrm{n} \times 1$ " vector $\mathrm{x}$ $(\mathrm{x}>0)$, makes $x^{T} C \leq 0$, then the Petri-net structure is limit.

(2) supposes it has only one " $\mathrm{n} \times 1$ " vector $\mathrm{x}$ $(\mathrm{x}>0)$, makes $x^{T} C=0$, then the Petri-net structure is conservation.

\section{II ).T_invariant}

One $\mathrm{T}$ _invariant is one " $\mathrm{m} \times 1$ " vector $\mathrm{y}(\mathrm{y} \geqslant 0)$, and meets condition:

$$
C y=0
$$

Suppose one transition sequence is activated ( the sequence count vector is " $\mathrm{u}$ "), token return from initial marking to initial marking, then based on formula (2):

$$
m_{0}=m_{0}+C u
$$

Concluded : $\quad \mathrm{Cu}=0$ Then " $\mathrm{u}$ " is one $\mathrm{T}$ invariant, namely " $\mathrm{y}=\mathrm{u}$ ", it denotes that element

(element $\mathrm{k} \geqslant 0$ )in $\mathrm{T}_{-}$invariant is times activated by transition in transition sets returning from $\mathrm{m} 0$ to $\mathrm{m} 0$. It is important applying validity of transition sets in $T_{\text {_ invariant to analyze }}$ activity of Petri-net structure .

Estimating Petri-net structure reliability to utilize limit and activity.

Petri-net invariant is not exclusive, calling invariant which is not other invariant linearity combination basic invariant.from linearity algebra, supposing that the order of matrix is $r=\operatorname{rank}(C)$, then the matrix has " $n-r$ " $P$ invariant and "m-r" $T$ invariant. The two invariants are attained by solving linearity equation $\mathrm{x}^{\mathrm{T}} \mathrm{C}=0$ and $\mathrm{Cy}=0$.

\section{ANALYSIS OF APPLYING PROBLEM}

Document management adapted to come true ,in OA(Office Automation) system, document is transferred from a dept to antother dept, which based on workflow technology. Sending document and receiving document are center of document management, utilizing workflow-net technology to realize document transferred is efficient.

\section{A. Workflow-net reliability analysis.}

Figure 5 is dispatch document manage workflow model, utilizing it to analyze workflow-net model construction 、 analysis and the same technology. 


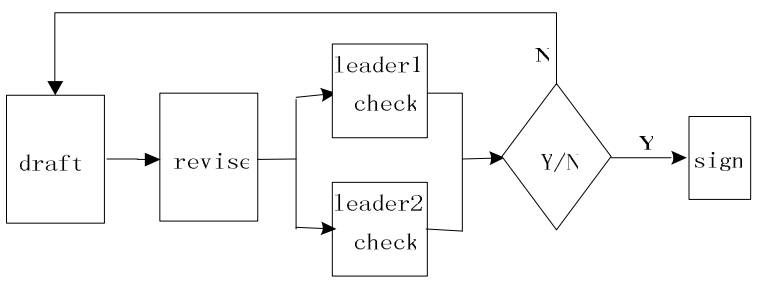

Figure 5 dispatch document workflow model

The flow process as follows: user drafts document, submitting, secretary transacts document with flow of serial structure and parallel structure based on sending document request. After Checking, transacting document has two choice: sign or draft.In all the flow process, administer can check and print flow process tracking table at any moment.

Figure 6 is workflow-net model of Figure 5. It includes six transition:draft、revise、leader 1 and leader 2 check、 auditing、 sign. Workflow process commences on token $\mathrm{p} 1$, and $p 8$ in the end, the role denotes transition in each process

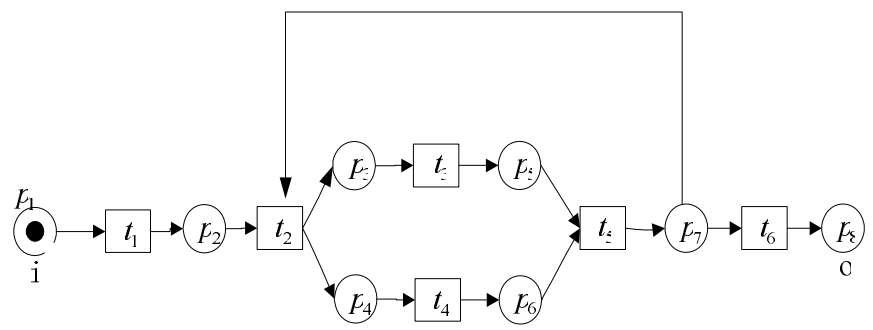

Figure 6 dispatch document workflow-net model

$t_{1}$.draft $t_{2}$.revise $t_{3}$.leader1 check $t_{4}$. Leader 2 check $t_{5}$. auditing $t_{6}$.sign

utilizing workflow-net definition to analyze model reliability:

(1) Increasing a transition $\mathrm{t}^{*}$ between Place $O$ and $i$, (namely ${ }^{\prime} t^{*}=\{0\}$ and $t^{*}=\{i\}$ ), then $w^{\prime}$ is a strong connectivity Petri-net.

(2) each node $(x \in P \cup T)$ belongs to path from $i$ to $O$

(3) no existing deadlock in model, each transition is executed.

The model is reliable from theory, in accord with workflow-net definition, however, in practice, the model has logic error: executing $t_{2}$, appearing and-join of Parallel structure . $\mathrm{t}_{2}$ has two input places, ${ }^{\prime} \mathrm{t}_{2}=\left(\mathrm{p}_{2}, \mathrm{p}_{7}\right)$, only existing tokens in $\mathrm{p}_{2}$ and $\mathrm{p}_{7}, \mathrm{t}_{2}$ can occur, the conclusion is not right. In practice, any one token exists in $\mathrm{p}_{2}$ or $\mathrm{p}_{7}$, namely drafting document is finished or auditing didn't pass, workflow should enter directily into revising state. The model is revised as Figure 7

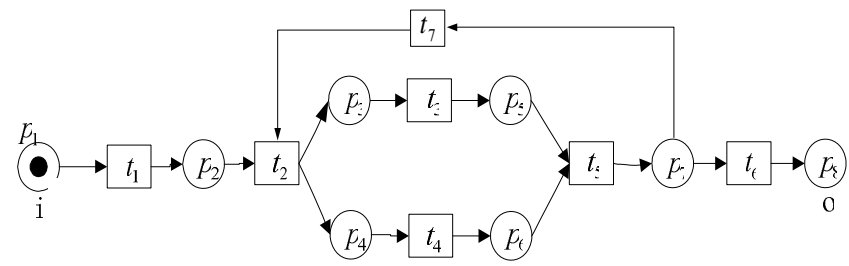

Figure 7 dispatch document workflow-net improvement model

Increasing a transition $t_{7}$ (return document), if transition $t_{5}$ is not past, the document will be returned $t_{2}$ via $t_{7}$, now , any one token exists in $\mathrm{p}_{2}$ or $\mathrm{p}_{7}, \mathrm{t}_{2}$ will occur, the improvement model (Figure 3)accords with not only Petri-net theory but sending document workflow in practice.

Further more, the improvement model is free choice Petri-net model. For any two transition of the improvement model $t_{1}$ and $t_{2}$, only existing ${ }^{*} t_{1} \cap{ }^{*} t_{2} \neq \phi$, then ${ }^{*} t_{1}={ }^{*} t_{2}$, for example , ${ }^{*} t_{3}={ }^{*} t_{4}={ }^{*} t_{7}$, the same as, the improvement model is proved as right structure via the analysis.

\section{B. Workflow-net invariant analysis method}

As follows, Resorting of invariant analysis method,for workflow model based on Petri-net, utilizing invariant analysis method, analyzing to accept document in document management, construct accept document management workflow model as Figure 8.

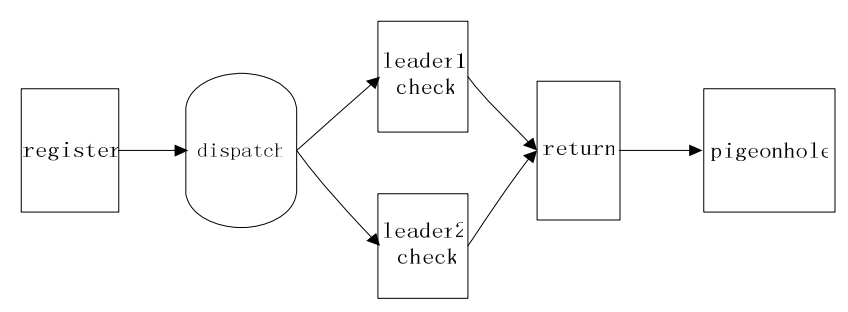

Figure 8 accept document management workflow model

Figure 9 is workflow-net model of Petri-net on figure 8. Workflow process commences on token $\mathrm{p}_{1}$, and $\mathrm{p}_{8}$ in the end, the role denotes transition in each process . $\mathrm{p}_{1}$ concludes a transacting document, equal to a token, at the same time $t_{1}$ consumes resource of $\mathrm{p}_{1} ; \mathrm{t}_{1}$ produces new token and puts it into $p_{2}$, then $t_{2}$ begins to run. If producing a token between $p_{3}$ and $\mathrm{p}_{4}, \mathrm{t}_{3}$ and $\mathrm{t}_{4}$ will execute at once, then $\mathrm{t}_{5}$ runs. $\mathrm{T}_{5}$ ensures parallel transition executing accurately. Producing to transacting document in $\mathrm{P}_{7}$, after $\mathrm{t}_{6}$ is pigeonholing, reach the lastest state $\mathrm{p}_{8}$.

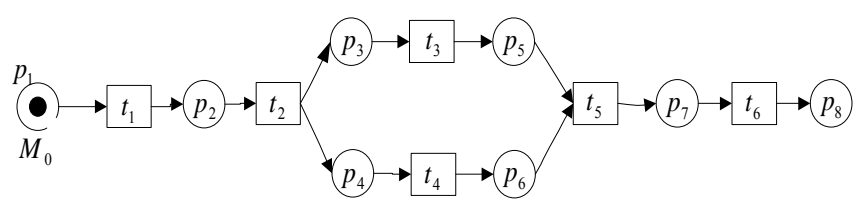

Figure 9 accept document Workflow-net model

The model(Figure 9) commences on $\mathrm{M}_{0}$, all likely states can attain in the reachable tree of model.Utilizing invariant 
analysis method and Workflow-net theory to analyze the performance of model .

\section{1) Workflow-net activity}

Workflow-net activity can be proved by constructing states equation, based on invariant analysis method. By formula $\mathrm{M}=\mathrm{M}_{0}+\mathrm{CU}$, supposing initial marking $\mathrm{M}_{0}=(1,0,0,0,0,0,0,0) \quad, \quad$ terminating marking $\mathrm{M}=(0,0,0,0,0,0,0,1)$, constructs the incidence matrix :

$C=\left[\begin{array}{cccccc}-1 & 0 & 0 & 0 & 0 & 0 \\ 1 & -1 & 0 & 0 & 0 & 0 \\ 0 & 1 & -1 & 0 & 0 & 0 \\ 0 & 1 & 0 & -1 & 0 & 0 \\ 0 & 0 & 1 & 0 & -1 & 0 \\ 0 & 0 & 0 & 1 & -1 & 0 \\ 0 & 0 & 0 & 0 & 1 & -1 \\ 0 & 0 & 0 & 0 & 0 & 1\end{array}\right]$

\section{$\because \quad(1,0,0,0,0,0,0,0,0)^{T}+C U=(0,0,0,0,0,0,0,1)^{T} \quad, \quad \therefore$}

$U=(1,1,1,1,1,1)$,pass transition sequence $T_{1} \cdots T_{6}$, all the workflow process is from initial marking to terminating marking, so the Workflow-net is active.

\section{2) Workflow-net boundary}

Boundary problem may transform states equation to solve problem. For model (Figure 9), $\because \exists Y>0, C Y \leq 0, \therefore Y=0$, so the Workflow-net is not boundary, the model should be improved. Improving model is as follows Figure 10.

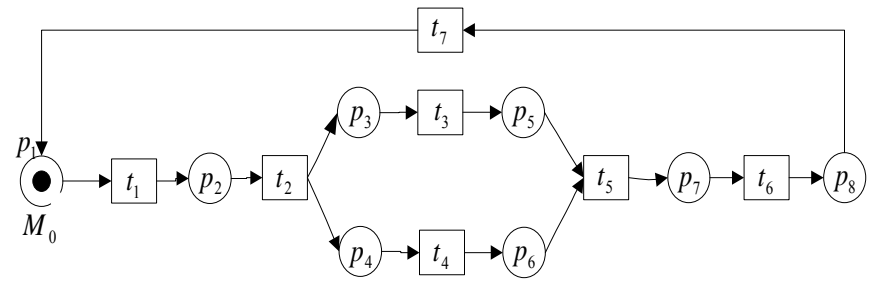

Figure 10 accept document Workflow-net improvement model

In improvement model(Figure 10), Increasing a transition $\mathrm{t}_{7}$, accept document process didn't execute one time ,but execute circularly.

constructs the incidence matrix over again:

$$
C=\left[\begin{array}{ccccccc}
-1 & 0 & 0 & 0 & 0 & 0 & 1 \\
1 & -1 & 0 & 0 & 0 & 0 & 0 \\
0 & 1 & -1 & 0 & 0 & 0 & 0 \\
0 & 1 & 0 & -1 & 0 & 0 & 0 \\
0 & 0 & 1 & 0 & -1 & 0 & 0 \\
0 & 0 & 0 & 1 & -1 & 0 & 0 \\
0 & 0 & 0 & 0 & 1 & -1 & 0 \\
0 & 0 & 0 & 0 & 0 & 1 & -1
\end{array}\right] ; C Y \leq 0 \Rightarrow\left\{\begin{array}{l}
-y_{1}+y_{7} \leq 0 \\
y_{1}-y_{2} \leq 0 \\
y_{2}-y_{3} \leq 0 \\
y_{2}-y_{4} \leq 0 \\
y_{3}-y_{5} \leq 0 \\
y_{4}-y_{5} \leq 0 \\
y_{5}-y_{6} \leq 0 \\
y_{6}-y_{7} \leq 0
\end{array}\right.
$$

$Y \geq 0$, so the improvement model is boundary.

\section{CONCLUSION}

This article introduced basic knowledge of Petri-net and Workflow-net, utilizing Workflow-net definitions and invariant analysis method to analyze Petri-net performance. In the complicated OA system, applying Workflow-net to construct model, applying reachable tree and incidence matrix method to analyze system feasibility reliability security ,etc. in practice, the methods have an better effect on Petri-net performance analysis. The methods make system project and performance optimal, more application of the technology demands to study and explore.

\section{REFERENCES}

[1] Girault C, Valk R. Petri Nets for Systems Engineering: A Guide to Modeling, Verification and Applications. Berlin: Springer-Verlag, 2003. 475-561

[2] Liu WD, Song JX, Lin C. Modeling and analysis of grid computing application based price timed Petri net. Acta Electronica Sinica, 2005, 33(8): 1417-1420(in chinese)

[3] WFMC-TC-1011. Workflow Management Coalition Terminology\&Glossary[S]

[4] Jang G W.K J, Kim Y Y. Integrated topology and shape optimitation software for compliant MEMS mechanism design[J]. Advances in Engineering Software, 2008, 39 (1) : 1-12

[5] Li J H, Gao S M, Liu Y S. Feature-based process layer modeling for surface micromachined MEMS [J]. Journal of Micromechanics and Microengineering, 2005,15 (3) : 620-630

[6] Girault C, Valk R Petri nets for Systems Engineering: A Guide to Modeling, Verification, and Applications. Berlin: Springer-Verlag, 2003 483-565

[7] Lerbet J. About the synchronization of MEMs [J].Nonlinear Analysis : Real World Applications, 2009,10(1) : 266-276

[8] Kim G H, Park K C. A continuum-based modeling of MEMS devices for estimating their resonant frequencies[J]. Computer Methods in Applied Mechanics and Engineering , 2008, 198(2) : 234-242

[9] YUAN Chongyi. The principle and application of Petri nets[M].Beijing : Publishing House of Electronics Industry, 2005.

[10] S Derisavi, H Hermanns, W H Sanders. Optimal state space lumping in markov chains[A]. Information Processing Letters[C]. Sept. 2003,87(6) : 311-315.

[11] Li Tao, Zhong Shisheng. Workflow model with colored timed Petri net and its performance analysis[J]. Journal of Computer-Aided Design \& Computer Graphics, 2006,18(6) : 821-831

[12] AALST W, HOFSTEDE A. YAWL : yet another workflow language[R]. Brisbane,Queensland,Australia : Queensland University of Technology, 2003

[13] R German. Iterative analysis of Markov regenerative models[J]. Journal of Performance Evaluation, 2001 